\title{
Factores climáticos y casos de Dengue en Montería, Colombia. 2003-2008
}

\author{
Climatic factors and cases of Dengue in Monteria, Colombia. 2003- \\ 2008
}

\author{
Alexander Cassab ${ }^{1}$, Víctor Morales ${ }^{2}$ y Salim Mattar ${ }^{1}$
}

\begin{abstract}
1 Facultad de Medicina Veterinaria, Instituto de Investigaciones Biológicas del Trópico, Universidad de Córdoba. Montería, Colombia.mattarsalim@hotmail.com

2. Departamento de Matemáticas y Estadística, Facultad de Ciencias. Universidad de Córdoba. Montería, Colombia.

Recibido 11 Agosto 2010/Enviado para Modificación 20 Noviembre 2010/Aceptado 8 Diciembre 2010
\end{abstract}

\section{RESUMEN}

Objetivo Evaluar la relación entre los casos de dengue y los factores climáticos pluviosidad (PLUV), humedad relativa (HUM) y la temperatura (TEMP) en el municipio de Montería en el periodo 2003 a 2008.

Métodos Estudio descriptivo, retrospectivo que relacionó tres variables climáticas y los casos de dengue en Montería. Se tomaron los promedios y medianas anuales y mensuales, se realizaron análisis de Shapiro-Wilk, coeficiente de correlación y análisis de varianza no paramétrica de Kruskal-Wallis, entre otros.

Resultados Los casos de dengue no presentaron una distribución normal ni diferencia significativa. Se registraron 1050 casos, en 2005 se reporto la cifra más alta con 305 y en 2004 solo 80 casos; el promedio anual fue de 175 . El análisis univariado de los casos dengue no estuvieron influenciados de forma significativa por la HUM relativa ( $\left.R^{2} 17 \%\right)$, la PLUV $\left(R^{2} 18,3 \%\right)$ y la TEMP $\left(R^{2} 1,8\right.$ $\%)$. Las variables PLUV y HUM relativa presentaron una alta correlación positiva de 0,798171. La PLUV y TEMP tuvieron un coeficiente de correlación negativa de 0,1310772 . La HUM relativa y la TEMP presentaron una correlación negativa de 0,6048030 . Las correlaciones más contundentes de casos de dengue vs clima se presentaron cuando se asociaron las tres variables conjuntas (TEMP, HUM y PLUV). Los fenómenos continentales del Niño y la Niña no influenciaron los casos de dengue.

Conclusiones Las asociaciones en conjunto entre la TEMP, HUM relativa y PLUV en la transmisión del dengue en Montería, son fuertes y consistentes a través del estudio retrospectivo realizado.

Palabras Clave: Clima tropical, Dengue, Colombia, prevalencia (fuente:DeCs, BIREME).

\section{ABSTRACT}

Objective Assessing the relationship between cases of dengue and climatic factors rainfall (PLUV), temperature (TEMP) and relative humidity (HUM) in Monteria during 2003-2008. 
Methods This was a descriptive, retrospective study related to climatic variables and dengue cases in Monteria. Rainfall (PLUV), relative humidity (HUM) and temperature (TEMP) data recorded annual and monthly medians and averages. The Shapiro-Wilks correlation coefficient and Kruskal-Wallis non-parametric variance tests were performed.

Results Dengue cases did not present normal distribution or significant difference. There were 1,050 cases; the highest number was reported in 2005 (305) but only 80 cases in 2004 (annual average was 175). Univariate analysis of dengue cases was not significantly influenced by HUM $\left(R^{2} 17 \%\right)$, PLUV $\left(R^{2} 18.3 \%\right)$ or TEMP $\left(R^{2}\right.$ $1.8 \%$ ). HUM and PLUV variables presented high positive correlation (0.798171). TEMP and PLUV had -0.1310772 negative correlation coefficient; TEMP and HUM had -0.6048030 negative correlation. The strongest climate-dengue case correlation was associated with combining the three variables (TEMP, HUM and PLUV). The Niño and Niña continental phenomena did not influence dengue cases. Conclusions This retrospective study showed a strong and consistent overall association between TEMP, HUM and PLUV affecting dengue transmission in Monteria.

Key Words: Tropical climate, dengue, Colombia, prevalence (source: MeSH, NLM).

$\mathrm{L}$ os cambios temporales y espaciales de las temperaturas, las precipitaciones y la humedad que, según las previsiones, tendrán lugar en los diferentes escenarios del cambio climático, afectaran la biología y ecología de los vectores y los huéspedes intermediarios y por consiguiente el riesgo de transmisión de enfermedades como el dengue $(1,2)$.

El objetivo de este trabajo consistió en evaluar la relación entre los casos de Dengue y los factores climáticos pluviosidad, humedad relativa y la temperatura en el municipio de Montería en el periodo comprendido de 2003 a 2008 .

\section{MÉTODOS}

Tipo de estudio

Se llevo a cabo un estudio de tipo descriptivo, retrospectivo e inferencial que buscó establecer de manera individual y asociada la relación entre variables climáticas y los casos de Dengue reportados en Montería durante el periodo comprendido de 2003 a 2008.

Población de estudio

La población de estudio correspondió a los pacientes registrados con dengue en los años 2003 a 2008 del municipio de Montería en la unidad de Vigilancia 
en Salud Publica de la Secretaria de Desarrollo de la Salud de Córdoba y en el Sistema de Vigilancia Epidemiológica SIVIGILA del Ministerio de la Protección Social.

Obtención de la información de los casos de dengue de Montería La unidad de Vigilancia en Salud Publica de la Secretaria de Desarrollo de la Salud de Córdoba, proporcionó la información de los casos de dengue registrados en Montería entre los años 2003 y 2004. El sistema de vigilancia epidemiológica SIVIGILA del ministerio de la Protección Social suministró los casos de dengue que se registraron ante esta entidad, entre los años 2005 al 2008.

Obtención de datos climatológicos

Se obtuvieron del Instituto de Hidrología, Meteorología y Estudios Ambientales (IDEAM) los registros históricos de precipitación, humedad y temperatura promedio de los años 2003 a 2008 de la estación meteorológica ubicada en Montería. Para observar los fenómenos del Niño y la Niña se uso la información disponible en el centro de predicción climática del National Oceanic and Atmospheric Administration of USA (3).

Municipio de Montería

El municipio de Montería es la capital del Departamento de Córdoba con una población de 378970 habitantes (4) está ubicado en la parte media del valle del río Sinú. Se localiza entre las coordenadas geográficas a los $8^{\circ} 45^{\prime} 27^{\prime \prime}$ de latitud Norte y los $75^{\circ} 53^{\prime} 34^{\prime \prime}$ de longitud al oeste de Greenwich, con una elevación de $18 \mathrm{msnm}$ y una extensión territorial del municipio de $3141 \mathrm{~km}$ (5).

Climatología de Montería. Se clasifica según el sistema de L. R. Holdridge como formación vegetal de bosque seco tropical, con una temperatura superior a $27^{\circ} \mathrm{C}$ y $1200 \mathrm{~mm}$ de precipitación y una humedad relativa de $84 \%$ (6).

Análisis de los resultados

Se implemento un análisis de Shapiro-Wilk para determinar si los datos cumplían con el principio de normalidad, de esta forma se podria escoger el modelo con mejor ajuste de acuerdo al supuesto. Se construyeron diagramas de cajas y gráficos de líneas a través de los cuales se pudo observar la presencia de datos atípicos y para describir las tendencias en el tiempo de las diferentes variables. Los datos se analizaron a través del coeficiente de 
correlación el cual fue organizado en una matriz de correlaciones. En ella se describió como están correlacionados los casos de dengue registrados en los diferentes años entre sí. Con este método se pudieron establecer asociaciones y diferencias de la variable dependiente entre los años del periodo de estudio y se pudo comparar si las causas que conllevaron a este comportamiento fueron similares. También se aplicó este método a las variables climáticas (variables independientes) para observar la influencia de las asociaciones sobre el modelo de regresión y si esto se expreso en los de casos de dengue. Finalmente, se aplicó el coeficiente de Pearson entre las variables meteorológicas y los casos de dengue para determinar el tipo de asociación que las variables compartían.

Dado que los datos de los casos de Dengue (variable respuesta) no se comportaron de forma normal (sin principio de normalidad estadística), se utilizo un análisis de varianza no paramétrica de Kruskla-Wallis, para determinar si existían diferencias significativas entre las medias de cada nivel del factor meses de los casos de dengue registrados para el municipio de Montería.

Es decir, se probó la hipótesis:

Ho: $\mu_{\text {enero }}=\mu_{\text {febrero }}=\ldots=\mu_{\text {diciembre }}$.

HA: al menos un promedio es distinto a los demás

Se tomaron para este análisis los promedios de cada mes a través de los seis de años de estudio como un nivel del factor.

Se describió de forma individual el comportamiento tanto de los casos de Dengue como cada una de las variables climáticas. Para ambos casos se tomaron los promedios y sus medianas con base en su comportamiento anual y en el periodo de estudio.

Para determinar el efecto de las variables explicativas (variables meteorológicas) sobre la variable respuesta (casos de dengue) se promediaron ambas a través de los seis años. Como la variable respuesta es una variable discreta que se formó por el número consecutivo de casos de dengue, entonces no resultó adecuado ajustar un modelo de regresión lineal múltiple usual. Esto debido a que se exige que la variable respuesta siga una distribución normal. En consecuencia, se utilizó un modelo de regresión de Poisson que es más adecuado para ajustar este tipo de datos. Se utilizó como medida de bondad de ajuste del modelo, el $\mathrm{R}^{2}$ en cual evalúa el porcentaje de variabilidad 
de la variable respuesta, que es explicado por las variables independientes. El modelo de Poisson aplicado fue el siguiente:

$\operatorname{Ln}(\lambda)=\beta \mathrm{o}+\beta_{1} X_{1}+\beta_{2} X_{2}+\beta_{3} X_{3}+\ldots+\beta_{k} X_{k}$.

Donde $\lambda=E(Y)$ representa el número promedio de eventos en una unidad de área o tiempo. Este modelo se basa en la distribución de probabilidad de una variable aleatoria Poisson que permitió integrar las diferentes variables en donde:

$\lambda$ : valor esperado de $\mathrm{Y}$

E: valor esperado

Y: casos de dengue promedio por meses (Variables dependientes)

$\mathrm{K}$ : corresponde al número de variables explicativas en el modelo.

Variables independientes:

$\mathrm{X}_{1}$ : pluviosidad en $\mathrm{mm}$ promedio mensual.

$\mathrm{X}_{2}$ : temperatura en grados centígrados promedio mensual.

$\mathrm{X}_{3}$ : humedad relativa promedio expresada en porcentaje.

Para el desarrollo del modelo se utilizaron algebra de matrices integradas al paquete estadístico "Excel" versión 2007 y "R" versión 2.10.0.

\section{RESULTADOS}

Los casos de dengue no presentaron una distribución normal ni diferencia significativa. Se registraron 1050 casos de dengue confirmados, en el 2005 se reporto la cifra más alta de casos de dengue con 305 casos y en el 2004 se registraron solo 80 casos de dengue, el promedio anual fue de 175 casos. En los meses de enero, febrero, marzo, abril, mayo y diciembre se presentaron los promedios más bajos en casos de dengue con 11,8 casos, 13,8 casos, 12,7 casos, 11,5 casos, 7,5 casos y 6,3 casos respectivamente (Figura 1). Los promedios más altos se registraron en los meses de junio, julio, agosto, septiembre, octubre y noviembre con 14 casos, 15,8 casos, 15 casos, 37,7 casos, 14,8 y 14 casos de dengue promedio mensual en Montería. El promedio de casos de Dengue en el periodo de estudio fue de 14,6 casos anuales.

Análisis de variables meteorológicas de Montería, 2003 -2008

Análisis de correlaciones de Pearson entre variables climáticas: Al observar los coeficientes de correlaciones entre las variables meteorológicas del 2003 al 2008 en la ciudad de Montería se encontró que la variable pluviosidad y humedad relativa presentaron una alta correlación positiva de 0,80 (Tabla 1). 
Figura 1. Comportamiento mensual en promedios de las variables durante el estudio. A. Pluviosidad vs casos. B. Humedad vs casos. C. Temperatura vs casos.

A

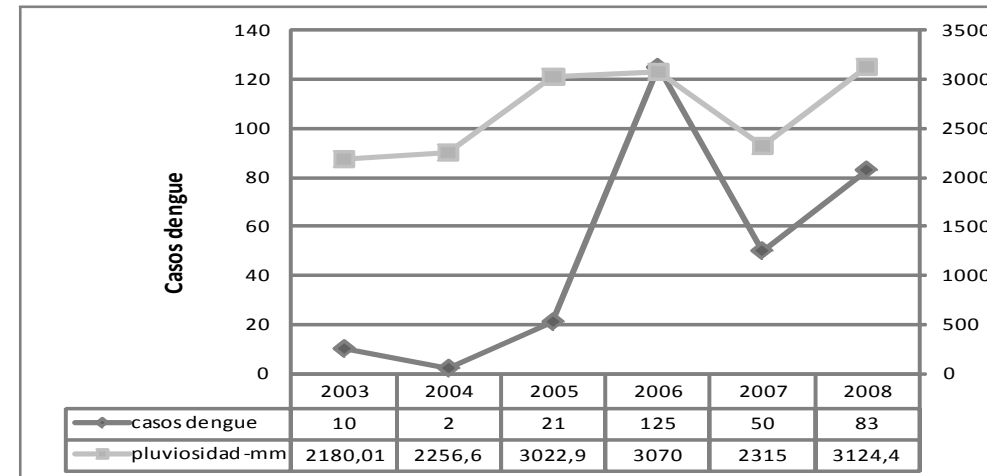

B

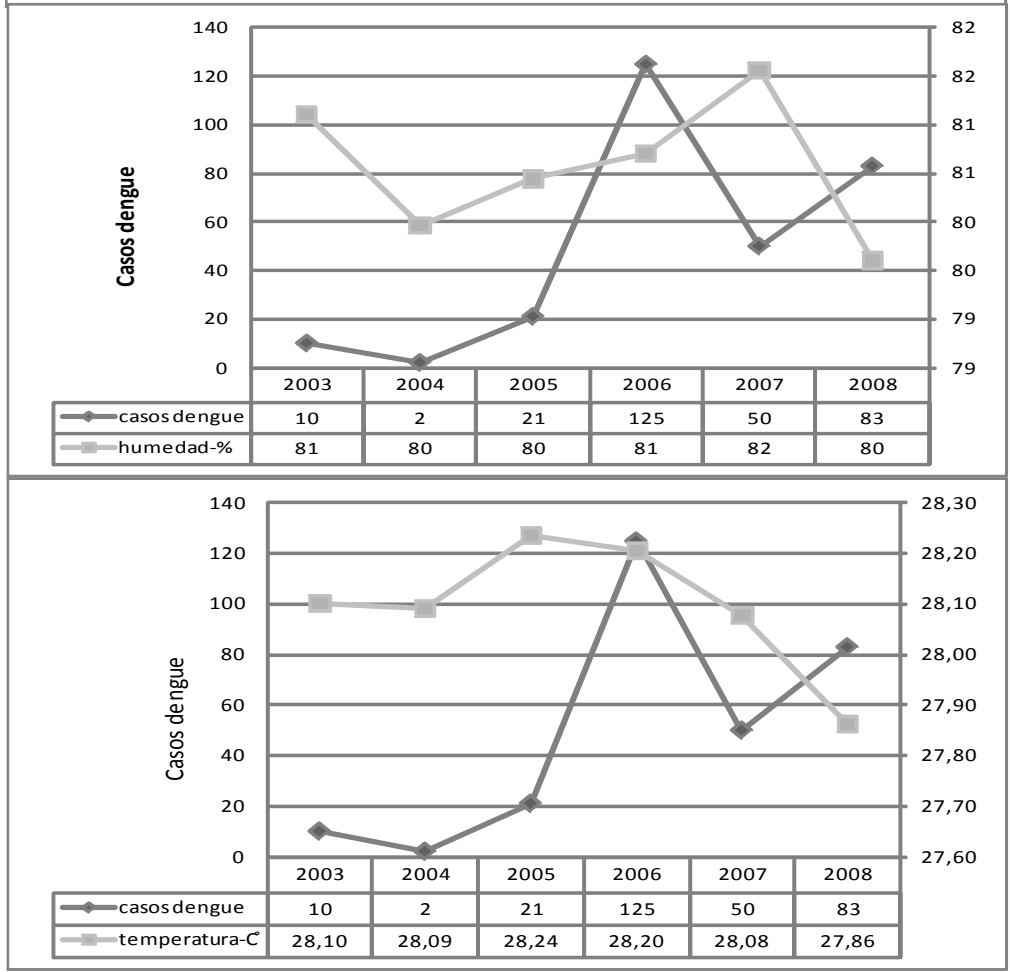

$\mathrm{C}$ 
Esto significa que las 2 variables estuvieron fuertemente asociadas entre si y que la pluviosidad y temperatura tuvieron un coeficiente de correlación negativa que fue de $-0,13$, lo que indica una débil asociación inversa entre estas variables (Tabla 1). La humedad relativa y la temperatura presentaron una correlación negativa de -0,60. Esta correlación negativa indicó una asociación inversa entre estas variables (Tabla 1). Las asociaciones encontradas entre las variables climáticas y los casos de dengue fueron positivas. Sin embargo, la pluviosidad fue la variable que presento la mayor asociación $(0,39)$ con respecto a la humedad relativa $(0,36)$ y la temperatura $(0,12)$.

Tabla 1. Matriz de correlación para las variables meteorológicas en Montería 2003-2008

\begin{tabular}{lccc}
\hline & Pluviosidad & Humedad relativa & Temperatura \\
\hline Pluviosidad & 1,00 & 0,80 & $-0,13$ \\
Humedad Relativa & 0,80 & 1,00 & $-0,60$ \\
Temperatura & $-0,13$ & $-0,60$ & 1,00 \\
\hline
\end{tabular}

Análisis descriptivo de la pluviosidad

Los meses en que se registró el menor promedio de precipitación fueron: enero (11 mm), febrero (11 mm), marzo (45mm), abril (96 mm), noviembre (111 mm), y diciembre $(85 \mathrm{~mm})$. Los meses que registraron los promedios $(134 \mathrm{~mm})$, agosto $(185 \mathrm{~mm})$, septiembre $(193 \mathrm{~mm})$ y octubre $(123 \mathrm{~mm})$ (Figura 1$)$. El promedio de precipitación en el periodo de estudio fue de $1168 \mathrm{~mm}$ anuales. Los años en donde se registraron las mayores precipitaciones fueron 2003 (1 485), 2005 (1 493) y 2008 (1 425). Las bajas pluviosidades se registraron en los años de 2004 (1 335), 2006 (1 294) y 2007 (1 238) (Figura 2).

Análisis descriptivo de la humedad relativa. En cuanto a la humedad relativa se halló que en los meses de agosto, septiembre y noviembre estuvo en 86,8 $\%, 87,4 \%$ y $86,6 \%$ respectivamente, siendo estos los promedios mas altos registrados. En los meses de enero, frebrero y marzo se notó una baja humedad relativa en Montería, con valores de 83,4\%, 82,8 \% y 81,4 respectivamente y un promedio anual de humedad relativa del 85,3 (Figura 1). El promedio de humedad relativa anual fue de $85,3 \%$ siendo 2004, 2007 y 2008 los años con humedad promedio anual más bajos 82,7 \%, 84,8 \% y $82,9 \%$ respectivamente, los años con mayores promedios fueron 2003, 2005 y 2006 con $87,2 \%, 85,8 \%$ y 88,5 respectivamente (Figura 2).

Análisis descriptivo de temperatura. En la Figura 1 se nota que las temperaturas promedio en Montería alcanzaron su promedio más alto en los 
meses de enero $\left(27,75^{\circ} \mathrm{C}\right)$, marzo, $\left(28,27^{\circ} \mathrm{C}\right)$, junio $\left(27,77^{\circ} \mathrm{C}\right)$ y julio $(27,72$ $\left.{ }^{\circ} \mathrm{C}\right)$. Los promedios más bajos se encontraron en los meses de febrero $(27,70$ $\left.{ }^{\circ} \mathrm{C}\right)$, abril $\left(27,68^{\circ} \mathrm{C}\right)$, mayo $\left(27,66^{\circ} \mathrm{C}\right)$, agosto $\left(27,73^{\circ} \mathrm{C}\right)$, septiembre $\left(27,74^{\circ} \mathrm{C}\right)$, octubre $\left(27,61^{\circ} \mathrm{C}\right)$, noviembre $\left(27,31^{\circ} \mathrm{C}\right)$ y diciembre $\left(27,48^{\circ} \mathrm{C}\right)$. El promedio de la temperatura fue de $27,7{ }^{\circ} \mathrm{C}$ siendo $2005\left(27,55^{\circ} \mathrm{C}\right), 2006\left(27,58^{\circ} \mathrm{C}\right)$ y $2008\left(27,46^{\circ} \mathrm{C}\right) \operatorname{los}$ años con temperatura promedio anual más bajos, los años con mayores promedios fueron $2003\left(27,96^{\circ} \mathrm{C}\right), 2004\left(28,02^{\circ} \mathrm{C}\right)$ y 2007 $\left(27,64^{\circ} \mathrm{C}\right)$ (Figura 2).

Ajuste del modelo para los casos de dengue en Montería. El análisis univariado de los casos dengue no estuvieron influenciados de forma significativa por la humedad relativa (R2 $17 \%$ ), la pluviosidad (R2 18,3\%) y la temperatura ( $\mathrm{R}^{2} 1,8 \%$ ) (Tabla 2$)$. En contraste, al analizar el modelo bivariado humedad relativa \& temperatura vs casos de dengue, se encontró de forma significativa que en un $39 \%$ se explicaban los casos. Otros modelos bi-variados no explicaron significativamente los casos de Dengue (Tabla 2).

En Montería, las correlaciones más contundentes de casos de dengue vs clima se presentaron cuando se asociaron las tres variables conjuntas (temperatura, humedad relativa y pluviosidad). Los fenómenos continentales del Niño y la Niña fueron débiles y moderados respectivamente y al parecer no influenciaron los casos de dengue estudiados (Tabla 2).

Tabla 2. Grado de relación entre las variables climáticas y los casos de dengue

\begin{tabular}{lc}
\hline \multicolumn{1}{c}{ Variables vs casos de Dengue } & $\mathrm{R}^{2} \%^{*}$ \\
\hline Pluviosidad & 18,3 \\
Humedad relativa & 17 \\
Temperatura & 1,8 \\
Pluviosidad y humedad relativa & 20 \\
Pluviosidad y temperatura & 22 \\
Humedad relativa y temperatura & 39 \\
Pluviosidad, humedad relativa y temperatura & 52 \\
\hline *Porcentaje en que los casos de dengue son explicados por las variables \\
meteorológicas
\end{tabular}


Figura 2. Comportamiento anual en promedios de las variables durante el estudio. A. Pluviosidad vs casos. B. Humedad vs casos. C. Temperatura vs casos

A

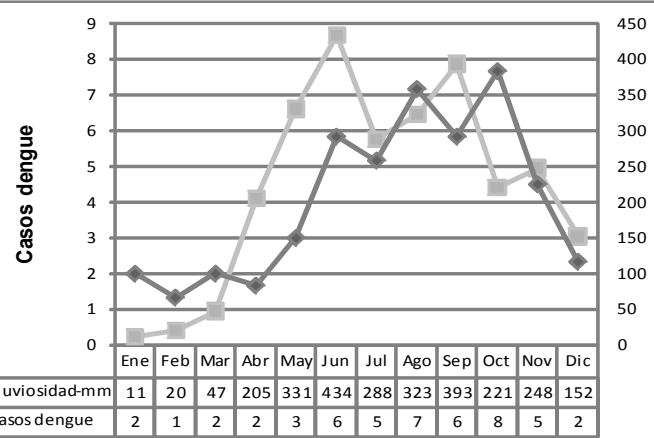

B

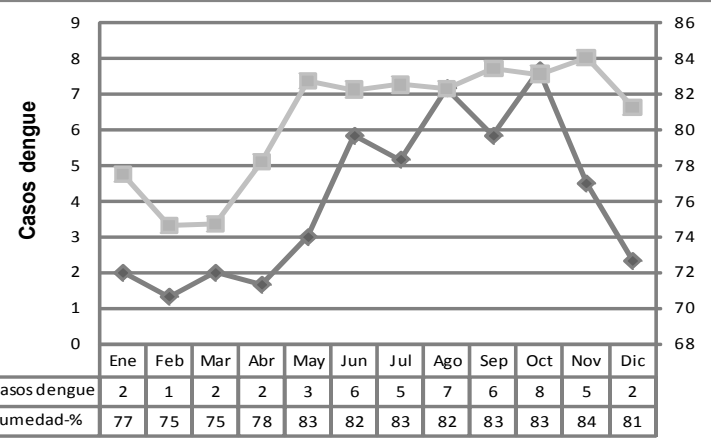

$\mathrm{C}$

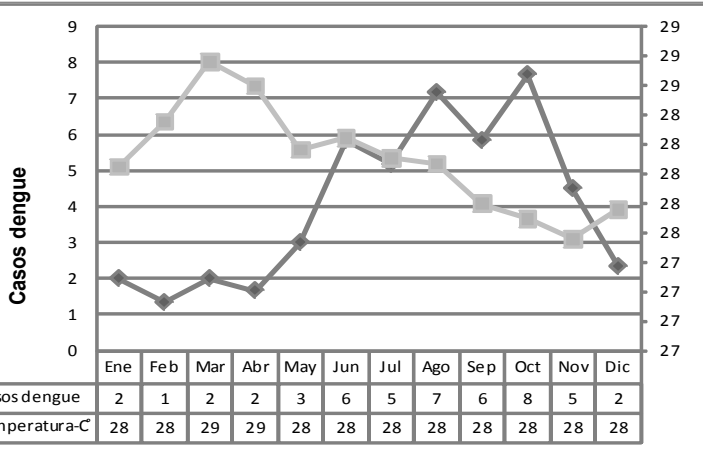




\section{DISCUSIÓN}

Los virus del dengue y su mosquito vector predominante Aedes aegypti, son endémicos en la mayoría de las regiones tropicales y subtropicales del mundo, donde causan epidemias estacionales importantes. El ciclo de transmisión puede estar influenciado por el clima, ya sea por el incremento de la temperatura y las precipitaciones, que pueden aumentar la población de Aedes aegypti. El incremento de la temperatura aumenta la tasa de desarrollo del vector, disminuye la duración de los ciclos de reproducción, estimula la eclosión de huevos y acorta el período de incubación del virus en el mosquito.

Son escasos los estudios y los modelos teóricos de la dinámica de la transmisión del dengue y la importancia de la temperatura, humedad y la precipitación, basados en la biología del mosquito para explicar los patrones de transmisión.

A escala mundial, varios estudios han puesto de relieve las características comunes de clima de las zonas donde la transmisión se produce. Mientras tanto, los estudios longitudinales de los datos empíricos han demostrado que la temperatura y la precipitación se correlacionan con la transmisión del dengue, pero no han demostrado la coherencia con respecto a sus funciones. Por ejemplo, la precipitación mensual acumulada y la temperatura media se correlacionaron positivamente con la transmisión del dengue en Tailandia. Sin embargo, fue el número de días de lluvia (independientemente de la cantidad) y la temperatura mínima que se asocio positivamente con la incidencia (7).

En nuestro trabajo se encontró una influencia importante entre el conjunto de variables climáticas (temperatura, humedad relativa y pluviosidad) y los casos de dengue. El uso de variables individuales no permitió explicar de forma contundente los casos de Dengue en Montería (Tablas 1 y 2). El asocio de las tres variables explicaron en un $52 \%$ los casos de Dengue (Tabla 2).

La temperatura promedio registrada en los municipios de Montería en los seis años de estudio fue de $27,7^{\circ} \mathrm{C}$. Septiembre, octubre y noviembre fueron los meses que compartieron promedios de temperaturas bajos. La temperatura promedio más alta fue en los meses de enero, marzo y junio (Figuras 1 y2). 
La temperatura no influyó en la presentación de los casos de Dengue en Montería. Esto debido a que estadísticamente el modelo de Poisson (temperatura vs casos) no explicó significativamente la ocurrencia de casos $(1,8 \%)$ (Tabla 2) ya que la explicación de asociación de causalidad requiere un valor $\geq 50 \%$. Resultados similares halló Johansson et al (8) en Puerto Rico con temperaturas promedios superiores $\left(30^{\circ} \mathrm{C}\right)$ a nuestra area de estudio y usando el mismo modelo de Poisson con datos de 77 municipios y una cohorte de 20 años de análisis. La ausencia de asociación, se podría explicar debido a que la temperatura óptima de transmisión y supervivencia del virus en el vector esta en el rango de temperaturas encontradas en el estudio de Puerto Rico y el nuestro. También podría deberse a que los datos en una escala de tiempo mes a mes no parecen demostrar que los años más cálidos (por ejemplo) muestran una asociación consistentemente con la prevalencia. Sin embargo, otros estudios sugieren que un aumento de un grado centígrado en la superficie del mar se refleja en un aumento del $20 \%$ de los casos de malaria manteniendo a las demás variables constantes (9). El hallazgo tiene sentido ecológico debido a que las temperaturas están acordes al comportamiento del vector en etapas en fase larval en el Caribe Cordobés.

Con respecto a la pluviosidad, los promedios más altos de precipitación fueron mayo, junio, agosto y septiembre y los meses con menores promedios fueron enero, febrero, marzo y diciembre (Figuras 1,2). El promedio anual de precipitación en el estudio fue de $1168 \mathrm{~mm}$ (Figura 1). La mayor precipitación del estudio fue de $1493 \mathrm{~mm}$ en 2005 (sin fenómeno de niño o niña) y la menor en 2007 (1 $238 \mathrm{~mm})$. Este último registro es contradictorio e interesante ya que en ese año se presento en forma moderada la niña donde se esperaría encontrar una mayor precipitación y un incremento de casos de dengue (Figura 2). Tampoco se explica cómo en el 2005 año meteorológicamente normal, (sin fenómeno de niño o niña) fue un periodo donde más se presentaron casos de dengue en Montería. Sin embargo, las condiciones pluviométricas en ese año fueron favorables para la proliferación de $A$. eagypti debido la precipitación (Figura 2).

Con respecto al fenómeno del Niño (2004 y 2006) se presentaron 80 casos en 2004 y 95 casos en 2006. En resumen, al analizar, los años del fenómeno del Niño $(2004,2006)$ y la Niña (2007) y los casos de dengue, no se encontró asociación (Tabla 3). Los resultados son similares a los trabajos en Puerto Rico y México, pero diferentes a los de Tailandia (10).

Otros hallazgos en Malasia, incluyeron un modelo biológico donde la precipitación fue un factor que permitió predecir de forma significativa los 
casos de dengue a principios de la temporada lluviosa. Sin embargo, no se asociaron con un cambio significativo en la abundancia de Aedes (11). En Indonesia, Malasia, Tailandia y la mayor parte del sudeste de las Islas asiáticas existe un aumento de la transmisión de la fiebre del dengue causados por la sequía que aumentan por el almacenamiento de agua alrededor de las casas que conduce a incrementar las poblaciones de Aedes aegypti y elevar la temperatura del aire lo que reduciría el período de incubación extrínseca para el virus en los mosquitos vectores y aumentar la capacidad del vector (12).

Nuestro estudio no tuvo en cuenta el índice larvario de Aedes, pero en el trópico del Caribe colombiano, algunas personas de bajos recursos económicos también almacenan agua en tanques sin protección. En Montería en épocas secas existen depósitos de aguas naturales (ciénagas, riachuelos y pozos superficiales o jagüeyes) que suplen de agua a la población humana y animales para sobrevivir sin mayor apuro.

Al contrario de algunas regiones de África que padecen sequías más extensas y periodos de lluvias cortos. La costa del Perú, Ecuador, Venezuela y Colombia tienen un aumento del riesgo de la malaria debido a la elevación de las poblaciones de vectores Anopheles que se desarrollan cuando los distintos tipos de hábitats inmaduros se inundan después de las fuertes lluvias tras un período de sequía (12). De igual forma, en el Noreste de Brasil las condiciones de sequía conllevan a un aumento de la fiebre del dengue y enfermedades respiratorias (12). No obstante, que esto podría extrapolarse al vector del dengue, el fenómeno del niño no pareció afectar los casos de dengue en nuestro trabajo.

Con relación a la humedad relativa, este trabajo halló que ligada a otra variable alcanza un porcentaje mayor de explicación que individualmente. En Montería por ejemplo, el modelo bi-variable con mayor grado de explicación se obtuvo cuando se integraron humedad relativa y temperatura (Tabla 3). Esto indica que la humedad relativa tiene importancia ecoepidemiológica cuando se incluye en modelos multi-variables. Análisis realizados en Tailandia de factores climáticos como la temperatura, la pluviosidad y la humedad relativa con la incidencia de dengue ha revelado que el dengue generalmente ocurrió cuando la pluviosidad fue comparativamente baja, la humedad relativa fue más alta que el promedio anual registrado y la temperatura se encontró por encima del promedio anual (13). 
En Colombia son escasos los estudios longitudinales sobre la ecología del vector que permitan elucidar y proyectar en la época de lluvias, transición y sequías que sucederá con el vector y la población de virus en él. En el trópico colombiano, no existen cambios climáticos extremos, usualmente se tiene una humedad relativa, temperatura y precipitaciones relativamente constantes, que al parecer no afectarían la dinámica del vector y por tanto la transmisión del dengue tendría una dinámica constante. Aunque el vector y el ciclo de transmisión son similares en todas las zonas endémicas, las relaciones descritas entre la transmisión y el clima son variables y en muchos casos sin explicación biológica alguna. Esto puede atribuirse entre otros factores a la heterogeneidad del clima subyacente, la vegetación (no estudiada por nosotros) y a las poblaciones de mosquitos, que podrían estar influenciadas por diferentes aspectos del medio ambiente. Otras debilidades de nuestro estudio es que no explican la movilidad de personas infectadas que podrían transportar diferentes serotipos de dengue, tampoco se analizo el índice de infestación ni el nivel social o índice de pobreza. Aunque el modelo bi-variable (humedad relativa vs temperatura) fue capaz de explicar la prevalencia de dengue, es muy probable que una casuística tan baja de casos de dengue dada por un alarmante subregistro, afectó el análisis de los datos y la asociación climática del Niño y la Niña.

En conclusión, el análisis de este trabajo muestra una línea tenue que reduce o minimiza los casos de dengues a simples números debido a la presentación anormal de la prevalencia observada en las zonas endémicas estudiadas sin duda afectadas por el subregistro. Sin embargo, las asociaciones en conjunto entre la temperatura, humedad relativa y precipitación en la transmisión del dengue en Montería, son fuertes y consistentes a través del estudio retrospectivo realizado. Estas asociaciones dependen de las características climáticas regionales y tienen una interpretación biológica, en contraste con los fenómenos continentales como el Niño y la Niña los cuales al parecer no estuvieron relacionados con la presencia de casos de dengue. El modelo aplicado en este trabajo podría servir a futuro a otros investigadores, siempre y cuando se tenga una casuística confiable.

Agradecimientos: A la Universidad de Córdoba, proyecto CIUC FMV 02-08. A la Secretaria de Salud de Córdoba por el suministro de los datos de los casos de Dengue entre los años 2003 y 2004. Al Ministerio de la Proteccion Social por la información del Sivigila. 


\section{REFERENCIAS}

1. Githeko AK, Lindsay SW, Confalonieri UE, Patz JA. Climate change and vector-borne diseases: A regional analysis [editorial]. Bulletin of the World Health Organization. 2000; 78(79):1136- 1147.

2. Turell MJ. Effects of environmental temperature on the vector competence of Aedes fowleri for Rift Valle fever virus. Research in Virology. 1989; 140: $147-154$.

3. National Oceanographic and Atmospheric Administration. [Internet]. Disponible en: http:// www.cpc.noaa.gov/products/analysis_monitoring/ensostuff/ensoyears.shtml. USA. Consultado en Marzo de 2009.

4. Departamento Nacional de Estadisticas [Internet]. Disponible en: http://190.25.231.242/ cgibin/RpWebEngine.exe/PortalAction?\&MODE=MAIN\&BASE=CG2005AMPLIADO\& MAIN=WebServerMain.inl Consultado en marzo de 2009.

5. Gobernación de Córdoba. [Internet]. Disponible en: http://www.cordoba.gov.co/ cordoba_municipios.html. Consultado: Marzo de 2009.

6. Corporación Autónoma Regional de los Valles del Sinú y del San Jorge-CVS, Corporación Nacional de Investigación y Fomento Forestal CONIF. Primera fase de la formulación del Plan General de Ordenación Forestal-PGOF. Departamento de Córdoba: CVS Convenio 027 de 2007. 2008; 1:126.

7. Promprou S, Jaroensutasinee M, Jaroensutasinee K [Internet]. Climatic factors affecting dengue hemorrhagic fever incidence in southern Thailand. Disponible en: http:// www.searo.who.int/LinkFiles/Dengue_Bulletins_Volumes_29_(2005)_CHAPTER05.pdf Consultado: Mayo de 2010.

8. Johansson MA, Dominici F, Glass GE. Local and global effects of climate on dengue transmission in Puerto Rico. PLoS Negl. Trop. Dis. 2009; 3(2): e382.

9. Gilma M, Oliveros H, Barnston G. The role of ENSO in understanding changes in Colombia's annual malaria burden by region, 1960-2006. Malaria Journal. 2009; 8:6.

10. Johansson MA, Cummings DAT, Glass GE. Multiyear climate variability and dengue-EI Niño southern oscillation, weather, and dengue incidence in Puerto Rico, Mexico, and Thailand: A longitudinal data analysis. PLoS Med. 2009; 6(11): e1000168.

11. Rogers D, Wilson A, Hay SI, Graham A. The global distribution of yellow Fever and dengue. Advances in Parasitology. 2006; 62: 181-220.

12. Anyamba A, Chretien JP, Small J, Tucker CJ, Linthicum KJ. Developing global climate anomalies suggest potential disease risks for 2006-2007. International Journal of Health Geographics. 2006; 5: 60.

13. Nakhapakorn K, Kumar T. An information value based analysis of physical and climatic factors affecting dengue fever and dengue hemorrhagic fever incidence. International Journal of Health Geographics. 2005, 4:13. 